\title{
An Empirical Study on Promoting Phonetic Teaching Through Smart phone-Based Mobile Learning \\ Xiao Jing ${ }^{1, a^{*}}$, Luo Yong ${ }^{2, b}$
}

${ }^{1}$ Foreign Languages Department of Zhuhai College Jilin University,

Zhuhai, Guangdong, China

${ }^{2}$ School of Electrical \& Information Engineering, Jinan University,

Zhuhai, Guangdong, China

axjangel@126.com, blyyong126@126.com

${ }^{*}$ Corresponding author

Key words: Smart Phones, Mobile Learning; Phonetic Teaching; Empirical Study

\begin{abstract}
Mobile learning, as a new digital learning model, improves teaching efficiency; and promotes the construction of informationized teaching platform. Taking phonetic teaching as an example, this paper investigates the promoting effect of smart phone-based mobile learning system on English pronunciation teaching. After comparative experiment, the statistics shows that integrating mobile learning into traditional phonetic teaching can effectively improve students' phonetic level; develops their phonological awareness and sense of language. Results from questionnaire and interviews indicate that first-year students lack a sufficient knowledge of mobile learning; before the activities, teachers' guidance is needed. During the process, teachers' remote monitoring, strategic instruction and psychological counseling are also necessary.
\end{abstract}

\section{基于智能手机的移动学习促进语音教学的实证研究}

\author{
肖婧 $1, \mathrm{a}^{*}$, 罗勇 $2, b$ \\ 1吉林大学珠海学院外语系, 珠海, 广东, 中国 \\ 2暨南大学电气信息学院, 珠海, 广东, 中国 \\ axjangel@126.com, blyyong126@126.com \\ 通讯作者
}

关键词: 智能手机, 移动学习, 语音教学, 实证研究

中文摘要: 移动学习作为一种新型数字化学习方式, 可以提高教学效率, 促进信息化教学平台 建设。本文以英语语音教学为例, 研究基于Android平台的移动学习系统对英语语音教学的促 进作用。历经十四周对比实验表明: 传统教学与移动学习相结合更能有效提高学生语音水平, 培养其语音意识与语感。问卷和访谈结果显示大一学生对移动学习认识不足, 在移动学习前 需要教师指导，过程中需要教师的远程监控、策略指导及心理疏导。

1. 引言

语言是传递人类思想和交流感情的重要工具; 语音作为语言的必要组成成分，也当属于 语言能力的范畴。[1]掌握一门语言准确的发音不仅可以运用它来准确表达思想, 也有利于捕 捉确切的信息。因此，语音教学在整个英语教学中有着举足轻重的作用。然而，纵观大学英 
语语音教学的现状, 一方面,语音在英语教学中被长期忽视; 另一方面, 传统课堂的诸多问 题导致语音教学效果不佳。2012 年教育部发布的《教育信息化十年发展规划(2011--2020年)》 指出要推进信息技术与教学融合, 建设智能化教学环境, 鼓励学生利用信息手段主动学习、 自主学习、合作学习。这份规划使我们看到将现代技术运用于教学中是未来教育发展的总体 趋势。

\section{2. 移动学习}

移动学习的研究最早始于美国，1994年卡内基梅隆大学开展了Wire Andrew研究项目，使 校内师生享受到网络时代移动学习所带来的便捷, 开创了这一研究的先河。目前移动学习没 有统一的概念，本文倾向于从技术和学习者两种方面来定义：即指以手持设备为媒介，可随 时随地进行的学习, 可以是正规学习, 也可以是非正规学习。移动学习模式尤其适合语言学习, 最早研究采用移动学习模式学习语言的是美国斯坦福大学。随后, 杜克大学和日本德岛大学 都开展了利用移动技术辅助外语教学的研究, 利用各种移动设备进行阅读、词汇、口语和听 力教学, 移动技术连接课堂内外, 取得了良好的效果。我国移动学习研究起步较晚, 但发展 迅速。2000年Desmond Keegan的报告《学习的未来: 从数字学习到移动学习》首次将这一概 念引入中国, 从此开启国内移动学习研究序幕。[2] 国内学者桂清扬最早指出: M-Learning 是继E-learning之后产生的一种新的远程学习方式, 是中国外语学习的未来。[3]

\section{3. 大学英语语音教学的现状及问题}

长期以来, 传统的英语专业教学模式以培养学生的语言能力作为重心, 讲授语音、语法、 词汇等系统语言知识, 忽视了学生的语用能力。在许多高校的专业课授课中, 教师的授课只 停留在语言形式的层面上, 要求学生反复掌握语法规则, 把语言视为一种静态的事物, 没有 让学生理解语言本质; 这就造成了许多学生虽然掌握大量语言知识, 但学生的语言交际能力 却未能得到有效的提高。在我国, 外语领域的口语测试也只是在最近十几年随着经济的发展 和对外交流的增多才被提到英语教学的议事日程上来; 起步较晚。英语专业的四级口试1994 年试点, 1999年正式向全国推行; 而专业八级口语测试2001年才开始实行; 或许是由于起步晚, 我国英语专业的口语测试中存在许多的问题。[4]

\section{4. 将手机移动学习融入语音教学}

\section{1 可行性分析}

中国手机网民的规模。CNNIC在京发布第36次《中国互联网络发展状况统计报告》显示, 截至2015年6月，中国网民规模达6.68亿，其中,手机网民规模5.94亿，网民上网设备中，手机 使用率达 $88.9 \%$, 继续保持上网第一大终端的地位。这些数据说明中国人, 其中包括许多大学 生更倾向于运用智能手机上网及处理事务, 这为智能手机成为移动学习工具, 支持英语教学 提供了硬件基础。智能手机的多项功能, 如: 下载学习软件。目前, 通过手机可以下载的英 语学习软件有许多, 类型也十分丰富: 音标学习软件、英语单词学习软件, 口语学习软件, 语法学习软件, 阅读学习软件, 英语游戏等。多媒体功能。智能手机支持拍照、录像、录音 和音频视频播放等媒体功能。学生可以利用录音功能把自己的口语练习过程录下, 通过软件 与范例进行对比。好的语音学习软件甚至可以即时对录音进行打分和提供及时反馈。远程沟 通功能。智能手机支持 $\mathrm{QQ}$, 微信等聊天工具, 以及微博等信息分享平台。教师可以利用微信 的语音短信功能发布通知, 发起话题讨论, 进行指导, 甚至可以监控学生学习进度。 


\section{2 研究对象与实验过程}

对象为珠海某高校电子信息专业一年级两个自然班, 共 67 人 (实验班 34 人, 对照班 33 人)。 实验班学生均下载安装语音训练软件“英语流利说”。对照班无要求。实施过程为 14 周。由于 没有设专门的语音课, 语音教学内容在英语口语课上完成, 每周两学时; 每次老师对语音知 识做一个专题培训, 约15分钟。前五周培训涉及音位发音, 后九周集中在超音位语音上, 即 重音(stress)、语调(intonation)、节奏(rhythm)、弱读(weak forms)、连读(linking)、减音(reductions) 等。两个班的教师及教学内容相同, 不同的是向实验班灌输移动学习理念; 详细介绍手机语 音训练软件的功能及使用步骤; 从第一周开始要求实验班利用该软件进行移动学习, 每周至 少三次, 每次时间累计不少于 20 分钟, 具体学习时间和地点由学生决定。并要求他们每周周 末将训练结果通过微信发给教师。

\section{3 研究工具}

4.3.1 语音试卷: 为了检验学习效果, 进行了实验前和实验后测试, 两套题题型和难度一 样。题目分四个部分： 20 个英标（元音10个, 辅音10个)，20个单词，10个句子，一篇短文。 由于语音评分具有一定主观性，考试由三位具有四六级口语考试考官资格的教师同时评分; 针对前测成绩进行了肯德尔和谐系数分析（Kendall's w =0.913）, 发现三位的评分一致性较 高。4.3.2 问卷: 这批学生学期末完成了一份调查问卷; 部分学生还接受了笔者访谈。问卷分 为两部分: 第一部分调查学生对语音学习的态度和看法, 第二部分调查学生对移动学习的认 识和看法。在正式调查前, 问卷进行试用, 根据反馈对问卷中测量项目进行了修正。

\section{5 结果与分析}

\section{1 两班班内与班间前后测试成绩对比}

从表 1 看, 两班前测平均分十分接近, 学期初语音程度在同一水平。后测显示两班相对前 测都有进步, 前后两次测试的 $\mathrm{T}$ 检验结果显示两班都有显著性差异, 可以说经过14周的语音学 习和训练, 两班都有明显的进步。（实验班 $\mathrm{t}=7.675$, 控制班 $\mathrm{t}=5.163$ ）。

表1 班内前测与后测 $\mathrm{T}$ 检验结果

\begin{tabular}{|l|l|l|l|l|c|c|}
\hline & \multicolumn{2}{|c|}{ 前测 } & \multicolumn{2}{c|}{ 后测 } & \multicolumn{2}{c|}{ 班内T检验 } \\
\hline 总人数 $=67$ & 平均分 & 标准差 & 平均分 & 标准差 & $\mathrm{t}$ & $\mathrm{p}$ \\
\hline 实验班 $=34$ & 64.35 & 6.332 & 85.71 & 5.824 & 7.675 & 0.000 \\
\hline 控制班 $=33$ & 63.88 & 7.873 & 76.52 & 6.869 & 5.163 & 0.000 \\
\hline
\end{tabular}

值得注意的是，表2显示实验班的后测成绩高于控制班，且两班之间在后测成绩上有显著 性的差异（ $\mathrm{t}=4.235 ）$, 也可以说虽然两班都有进步, 但实验班同学学习更加有效果, 进步更 为明显。可以得出这样的结论: 以教师为主导的传统语音知识讲解, 结合借助手机软件移动 学习后的英语语音训练方式能更有效地提高大学生的英语发音质量。

表2 班间后测 $\mathrm{T}$ 检验结果

\begin{tabular}{|l|l|l|c|c|}
\hline & \multicolumn{2}{|c|}{ 后测试 } & \multicolumn{2}{c|}{ 组间 $\mathrm{T}$ 检验 } \\
\hline 总人数 $=67$ & 平均分 & 标准差 & $\mathrm{t}$ & $\mathrm{p}$ \\
\hline 实验班 $=34$ & 85.71 & 5.824 & \multirow{2}{*}{4.235} & 0.000 \\
\hline 控制班 $=33$ & 76.52 & 6.869 & & \\
\hline
\end{tabular}

\section{2 实验班高分与低分组移动学习次数与时间对比}

通过对学生每周微信反馈信息 (学习次数及时间) 的统计, 发现实验班高分组 4 名同学与 低分组的 4 名同学进行移动学习的次数上有差异。表 3 显示: 高分组同学每周平均训练 9.5 次, 
每次训练时间达到 21.5 分钟; 而低分组的同学平均每周训练约 2.75 次，每次 18.5 分钟，仅达到 老师的基本要求。很显然高分组每周用在语音训练上的时间比低分组要多。这也验证了有学 者提出的语音训练的程度对学习效果会产生影响, 语音成功者比不成功者接受的语音训练更 多。

表3 高分与低分组每周平均学习次数及每次平均时间

\begin{tabular}{|l|c|c|c|}
\hline & 后测平均分 & 每周平均次数 & 每次平均时间 \\
\hline 高分组 & 92.5 & 9.50 次 & 21.5 分钟 \\
\hline 低分组 & 71.5 & 2.75 次 & 18.5 分钟 \\
\hline
\end{tabular}

\section{6. 问卷调查结果与分析}

问卷发放67份，全部收回，均有效。根据问卷和访谈结果，得出以下结论：

\section{1 大一新生对移动学习了解程度较低。}

问卷显示只有 $45 \%$ 的同学在进入大学前, 听过“移动学习”这个学习概念并对此有所了解。 这一结果比王伟等[5]的调查结果要稍好一些, 他们的调查中 $98 \%$ 学生第一次听“移动学习”这 一概念。两次调查相距五年, 学生在这段时间对移动学习的认识有所提高。访谈中我们了解 到高中应试教育影响较大, 手机、手提电脑及掌上电脑被认为会分散注意力, 在高中不提倡 使用。因此针对大一同学, 教师开展移动学习活动前, 应该全面介绍相关理念, 并对移动学 习策略和方法进行培训。

\section{2 对移动学习的热情高, 认为对语音学习有促进作用。}

问卷中有 $93 \%$ 的同学, 其中包括对照组的同学, 表示对移动学习感兴趣, 认为对语音学 习有促进作用。通过访谈了解到对照班同学虽然这次没有加入到语音移动学习的活动中, 但 大部分表示如果有机会很愿意参与。实验班的同学们表示移动学习减缓了他们在传统课堂出 现的焦虑和紧张的情绪, 增加了他们大声大胆练习语音的机会, $82 \%$ 的同学认为传统教学方 式与移动学习相结合, 效率事半功倍。

\section{3 对于新生, 移动学习过程中需要老师的监控和指导。}

问卷结果表明 $87 \%$ 的学生认为移动学习需要老师的监控。访谈中了解到实验班大部分学 生认为每周发微信向老师汇报学习情况很有必要, 对学习有督促作用。低分组四位同学一致 认为这种做法对于他们尤为重要, 因为学习自主性较差。因此, 移动学习过程中, 教师应利 用现代技术对学生学习进行远程的监控和指导, 以确保移动学习具有一定的规律性和连续性, 学生注意力集中, 知识印象清晰; 如出现焦虑的情绪, 教师应适时针对个别学生作有效的学 习策略指导与心理方面的疏导。

\section{References}

[1] Gimson, A. C.An Introduction to the Pronunciation of English. London: Edward. 1989. pp $15-16$

[2] Keegan, D. The future of learning: from E-learning to M-learning. DOI:http://learning.ericsson.net/Leonardo/book.2000.

[3] Gui Q.Y. M-learning---the future of China's foreign language teaching.Computer-assisted Foreign Language Education. 2003.

[4] C. Jie. Reflections on the reform of oral English test. Heilongjiang Researches on Higher Education .2009, vol.3. 62-63. 
[5] Wang W, Zhong S.C \& Lu S.L An empirical study on the mobile learning of college students. Open Education Research 2009. (12) 81--86 